\title{
Infrared thermography applied to the validation of thermal simulation of high- luminance LED used in automotive front lighting
}

\author{
by C. Rongier*,**, R. Gilblas*, Y. Le Maoult*, L. Redjem-Saad** and F. Schmidt* \\ * Université de Toulouse, IMT Mines Albi (Institut Clément Ader), Campus Jarlard, F-81013, Albi cedex 09 France \\ ${ }^{* *}$ Valeo Lighting System, 34 rue Saint André, 93102 Bobigny, France
}

Automotive front lighting is evolving towards digital and adaptive high definition beams. To create such functions, multiple LED designs are replaced with new LED concepts using only one high luminance LED. Light concentration emitted from this optoelectronic component generates a high density of energy which must be thermally managed. Indeed, optical performance and reliability of components are directly linked to the LED temperature. Thus, to optimize cooling system, accurate and efficient numerical models must be developed. The validation of these models are based on comparison with experimental data. In this paper, an infrared camera was used to measure quantitatively the temperature of a high luminance LED emitting area and was then compared to 3D thermal simulations.

\section{Introduction}

The thermal management of high power LED systems is required in order to ensure both optical performance and LED reliability [1]. Therefore, the knowledge of temperature distribution on LED active region (or emitting area) is needed to determine the junction temperature of such optoelectronic component. Different studies have investigated the use of infrared thermography in order to obtain a quantitative heat distribution dissipated by LED [2, 3]. However, this technique is limited by the knowledge of the emissivity of LED active region [4,5]. Moreover, the multilayer structure as well as the semi-transparent behaviour of such an active component reinforces the challenges regarding quantitative thermal measurement.

\section{Preliminary experiments}

Preliminary experiments have consisted in optical characterization of LED emitting area. Thus, a Vertex Brucker IRTF spectrometer equipped with separators has been used, respectively for accessing to LED spectra in near [0.8-2.5] and medium infrared [3-20] $\mu \mathrm{m}$ spectral band. This characterization was performed using a goniometer and assuming lambertian emission, due to LED emitting area dimensions. The emissivity spectrum of the active region is needed in order to determine the adaptive infrared camera and avoid calibration issues. Figure 1 shows emitting area spectra obtained for two components of high luminance LED. It can be seen that a quite reliable values of emissivity is obtained from 1 to 10 microns, since in this spectral region, the emitting area has the same behaviour as a gray body, with an emissivity of about 0.9. However, in the spectral band [10-20] microns, emissivity reaches a minimum and then a maximum value that corresponds to an absorption band on the active region, due to the polymer resin, probably. Therefore, to use IR thermography, the choice of the spectral band of the IR camera as well as the using of a macro lens is fundamental to obtain an accurate measurement, while ensuring a good spatial resolution.

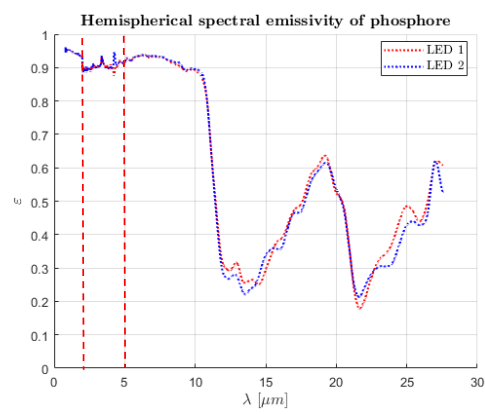

Fig. 1. Hemispherical emissivity spectrum of high luminance LEDs emitting area.

\section{Experimental set up}

An experiment device has been developed to measure the temperature of high luminance LED's active region. An infrared camera FLIR SC7000 equipped with a microscope lens G1 is positioned in front of the LED, mounted on its 
cooling system. Both objective choice and calibration step are described in this paper. The temperature is also monitored using thermocouples. Once the LED temperature is stabilized, a surface temperature distribution measured using IR thermography is obtained in order to determine the temperature distribution at the surface of the LED.

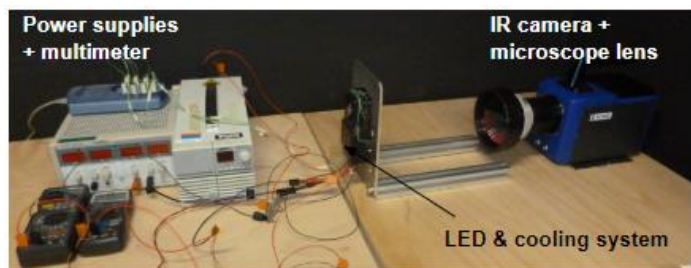

Fig. 2. Experimental set up for high luminance LED infrared thermography.

\section{Confrontation with 3D heat transfer simulation}

A 3D heat transfer model of high luminance LED has been developed. In order to model accurately the different layers that compose the LED structure, the 3D geometry of the LED is obtained using SEM images. Then, based on the optical and thermal energies balance, the simulation set up has been possible. Previously, optical measurement was made on LED in order to determine the optical flux as well as LED spectrum and angular distribution for each configurations. Once measurement and simulation have been performed, temperature distribution on high luminance LED can be investigated, as shown on fig. 3. Thus, temperature profile on the active region have been compared for $\mathrm{I}=2 \mathrm{~A}$ case (fig. 4).

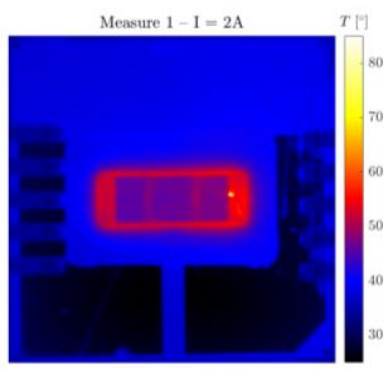

(a)

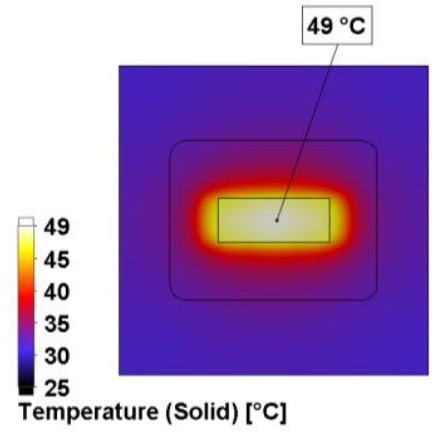

(b)

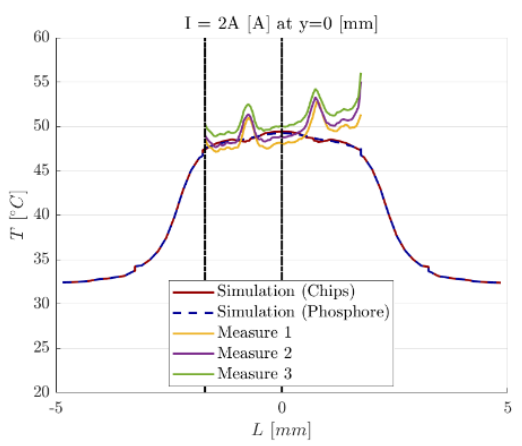

(c)

Fig. 3. Temperature distribution on high luminance $L E D$ - (a) IR thermography, (b) $3 D$ thermal simulation. - Temperature profiles on high luminance $L E D$ on the active region at $y=0 \mathrm{~mm}(c)$

According to fig. 3b), it can be seen that the simulation predicts no deviation between the active region (called phosphore) and the chip. Therefore, knowing the emitting area temperature distribution, the junction temperature of the LED is determined. Then, by comparing measured profiles with computed ones, several differences can be identified. Three region are in good agreement with simulated results: they corresponds to the LED chips. However, four peaks can be seen as well. The increase of temperature related to those peaks is induced by a change of material: the emitting area is protected by a specific layer whose emissivity is different from the one of active region. Therefore, since IR camera is calibrated with the emissivity of the emitting area, a material change generates differences between numerical simulation and measurement.

\section{REFERENCES}

[1] Massol, L., "Les LEDs pour l'éclairage - Fonctionnement et performances, critères de choix et mise en œuvre, $2^{\mathrm{e}}$ Edition, Dunod, 2015 (in french)

[2] Belfio, H., Veneaub, J.F. and Fudyma, O., "Chip emissivity mapping for blue and white light emitting diodes". $12^{\text {th }}$ International conference on Quantitative Infrared Thermography, 2014.

[3] Lee, D., Choi, H., Jeong, S., Jeon, C.H., Lee, D., Lim, J., Byon, C., Choi, J., "A study on the measurement and prediction of LED junction temperature. International Journal of Heat and Mass Transfer - Vol. 127, pp. 12431252,2018

[4] Jang, H., Lee, J.H., Byon, C., Lee, B.J., Innovative analytic and experimental methods for thermal management of SMD-type LED chips. "International journal of Heat and Mass transfer - Vol. 124, pp. 36-45, 2018.

[5] Corfa,C., Gasse, A., Bernabe, S., Ribot, H., Analytical and FEM simulations of the thermal spreading effect in LED modules and IR thermography validation. "2010 11th International Thermal, Mechanical \& Multi-Physics Simulation, and Experiments in Microelectronics and Microsystems (EuroSimE)" - pp. 1-8, 2010. 